\title{
Interacting With Anatomically Complete Robots
}

\author{
A Discussion About Human-Robot Relationships
}

\author{
Christoph Bartneck \\ University of Canterbury \\ Christchurch, New Zealand \\ christoph.bartneck@canterbury.ac.nz
}

\begin{abstract}
Abyss Creations LLC is selling highly realistic and anatomically complete dolls for decades. The interaction that customers have with the dolls has been subject to many controversial discussions. David Levy already showed that humanity has been rather inventive when it comes to machines that useful for sexual purposes. Abyss Creations recently revealed its Harmony platform that consists of a robotic head that is attached to their doll bodies. This paper present an interview with Matthew McMullen, CEO and Creative director of Abyss Creations about the Harmony platform and its implications on human-robot relationships.
\end{abstract}

\section{CCS CONCEPTS}

- Human-centered computing $\rightarrow$ Human computer interaction (HCI);

\section{KEYWORDS}

companionship, robot, doll, relationship, intimacy

\section{ACM Reference Format:}

Christoph Bartneck and Matthew McMullen. 2018. Interacting With Anatomically Complete Robots: A Discussion About Human-Robot Relationships. In HRI '18 Companion: 2018 ACM/IEEE International Conference on HumanRobot Interaction Companion, March 5-8, 2018, Chicago, IL, USA. ACM, New York, NY, USA, 4 pages. https://doi.org/10.1145/3173386.3173387

Before entering the more philosophical discussion around human machine relationships I would like to ask some more practical questions. The Real Dolls seem highly customizable (see Figure 1). How many different configurations are you offering? What are the most popular choices? Female dolls seem to dominate your website, are most of your customers male?

The dolls are all configurable, so there aren't really a number of configurations. Clients choose a body, a face, and then skin tone, nail finish, makeup (individual choice for lips, eyes, etc.) and then hairstyle. There are thousands of combinations possible. Yes, most of our clients are male, about $80 \%$. The remaining $20 \%$ are either female or couples. $80 \%$ of the dolls we make are female.

Permission to make digital or hard copies of all or part of this work for personal or classroom use is granted without fee provided that copies are not made or distributed for profit or commercial advantage and that copies bear this notice and the full citation on the first page. Copyrights for components of this work owned by others than the author(s) must be honored. Abstracting with credit is permitted. To copy otherwise, or republish, to post on servers or to redistribute to lists, requires prior specific permission and/or a fee. Request permissions from permissions@acm.org.

HRI '18 Companion, March 5-8, 2018, Chicago, IL, USA

(C) 2018 Copyright held by the owner/author(s). Publication rights licensed to Association for Computing Machinery.

ACM ISBN 978-1-4503-5615-2/18/03 .\$15.00

https://doi.org/10.1145/3173386.3173387
Matthew McMullen

Abyss Creations LLC

San Marcos, California

matthewmcmullen@realdoll.com

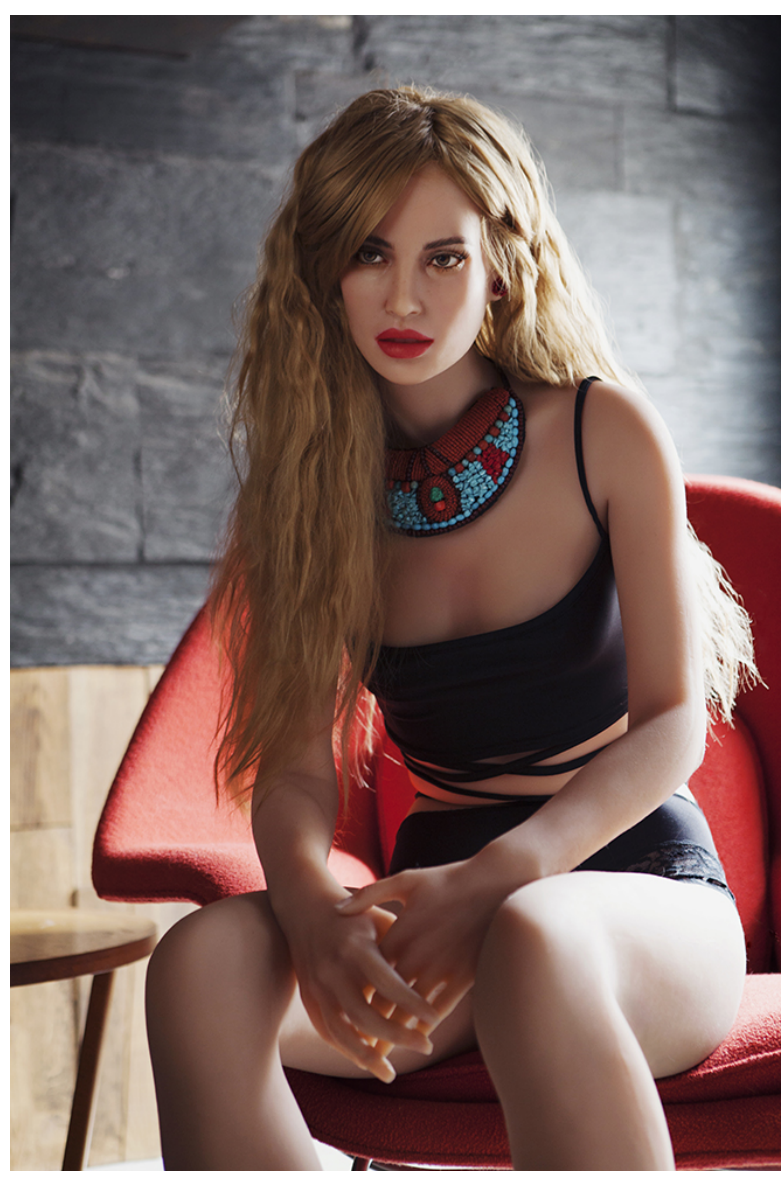

Figure 1: A Real Doll

You started to offer a new robot that is build upon Real Dolls with your company Realbotix. You started to animate Real Dolls' face. Why did you start with animating the face and not with any other body part?

We wanted to add personality, expression, rather than focusing on sexual gyrations etc. This is more about companionship than sex, and is meant to augment the aesthetic of the dolls rather than distract from it.

What degrees of freedom does the head have?

There are 10 DOF including neck pitch and yaw, jaw open/close, smile/frown, eyes pitch and yaw, eyelids open/close, and eyebrows up/down (see Figure 2). 


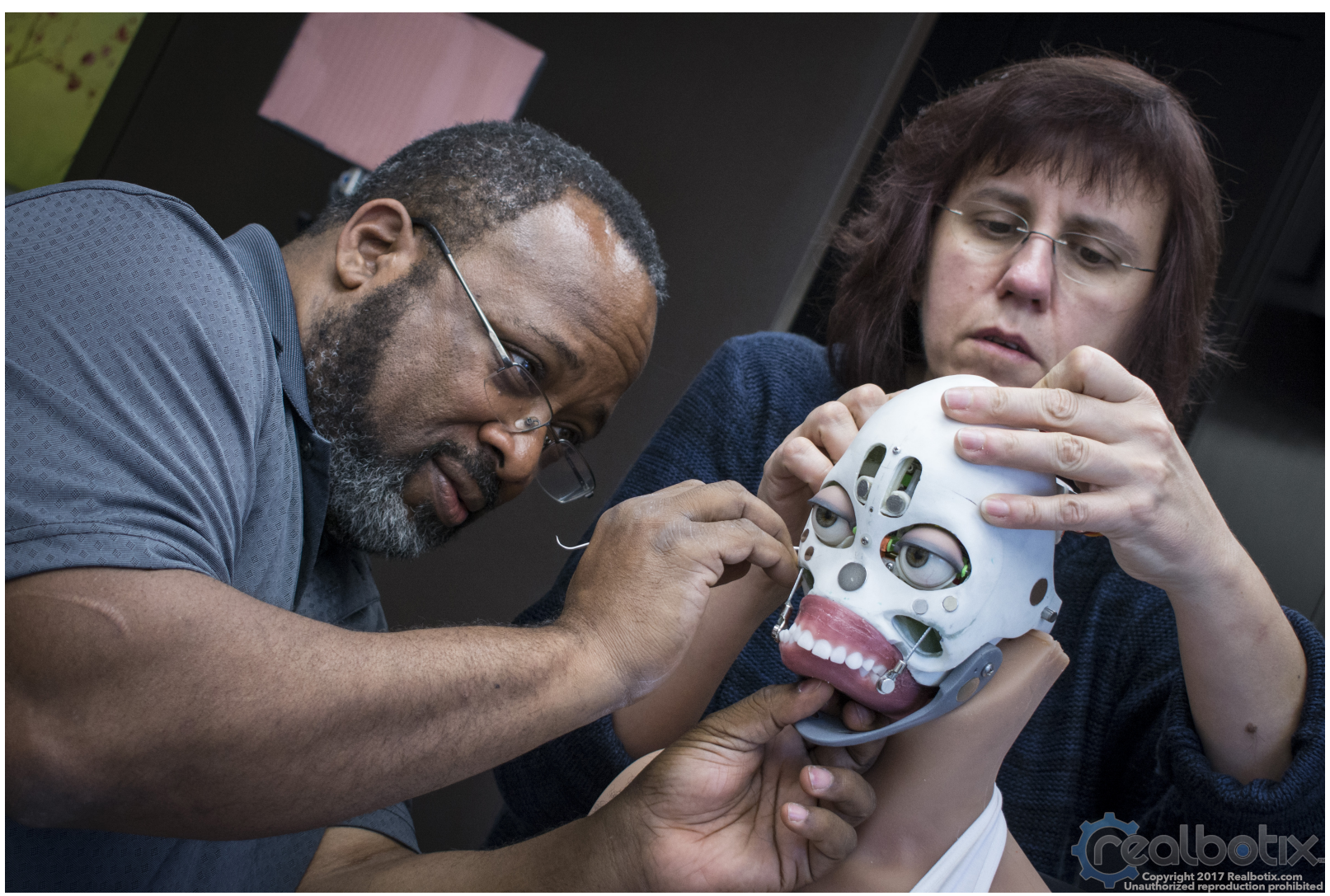

Figure 2: Assembly of the robotic head

You are connecting the Real Doll Robot, called Harmony (see Figure 3) to a mobile app and to a VR environment. Could you in short explain how these different components will work together?

The App is the core of the system. It is a user customizable AI with multiple personality traits available. Different combinations of traits will yield different interactions and experiences when talking to the generated AI. This AI can then be used in to connect to the robotic system, or if so desired, a VR and/or AR system once development is complete.

Will this Real Doll Robot Platform be available for academic research? Are you intending to offer an open API?

Yes, we are planning to offer both an SDK for the AI and a Hardware development kit for the robot's face options.

I assume that your customers' needs and desires span a considerable spectrum. Can you share some of the more common patterns of people interacting with the dolls? What relationships do they form with their dolls? Will the interaction with the robot be different from the one with a doll?

Most of the people who own dolls are drawn to them because they want some form of companionship, or the feeling/simulation of such. Sexual attraction may very well be part of that equation initially, but it seems many begin to view the doll as something more over time, and even imagine personalities or characters for them. The AI and robotic platform is meant to help facilitate that aspect for those that are interested in it.
If the imagination of the user is such an important part for the interaction, would it not be better to keep the empty canvas of the doll instead of providing explicit behaviour, similar to how reading books often surpasses watching its movie adaptation?

Everyone has varying degrees of imagination. That's why some prefer movies to books. Having an actual conversation with a robot is very different from imagining that such a thing is occurring.

Is a relationship with a doll or a robot different from a relationship to a human? Do the dolls represent a human or are they of a completely different quality? Are the users having a relationship with a doll or with the representation of a human?

The robot is not meant to replace a human. It is not supposed to pass as one, or fool anyone into thinking it is human. It is an entirely new form of companionship. To varying degrees, I am sure that there will be aspects of imagination with owners of the robots, much as there are with the dolls themselves. So yes, the short answer is that relationships with dolls or robots will be a whole new type of relationship.

Could interaction with a Real Doll (Robot) be considered a form of therapy? Are Real Doll (Robots) being used for any kind of therapy in hospitals or other health organisations?

We absolutely feel this technology could have far reaching potential for many types of therapy. AI equipped human-like robots can have infinite patience, repeat themselves over and over, and can provide needed reminders and alerts in a more personal way than 
say a computer of other smart device. The human-like appearance will prove to be a valuable aspect to these potential uses.

The Paro Seal robot [2] is being used in elderly care as a companion robot. Although not sexual in nature, it certainly mirrors Real Dolls' function as a companion. The question remains if we fulfil the users' desire for companionship not with just another form of loneliness. Should the goal not be to have lonely people interact with real humans?

The assumption there is that all people have an equal capacity and desire to interact with other humans. It should be considered that some do not have that capacity, or do not want it.

When interacting with a doll, the user is in complete control of the situation. Does this security of control offer the safety that some people need when interacting with "others"?

Perhaps. In some cases. But there are certainly deeper psychological and philosophical questions that could need to be evaluated to completely answer this.

Kathleen Richardson started the Campaign Against Sex Robots ${ }^{1}$ and most of her arguments are based on the idea that having sex with a female robot degrades women and may even lead to an increase in false expectations, similar to how the pornographic industry has lead to an unrealistic expectation of how fast a plumber arrives at your doorstep after a call for help. Does sex with a Real Doll degrade women (or men) and is there any evidence that this may result in an increase of inappropriate behaviour towards real woman and men? Have any of your dolls ever be returned for repair that showed signs of abusive behaviour towards them?

No. In fact, most of our clients treat their dolls with amazing levels of care. I have personally not observed anything whatsoever to suggest that this type of thinking is supported by any data. Perhaps a study could be done prior to making such broad assumptions?

Robert Sparrow in his paper "Robots, Rape, and Representation" [1] argues that:

... the design of realistic female robots that could explicitly refuse consent to sex in order to facilitate rape fantasy would be unethical because sex with robots in these circumstances is a representation of the rape of a woman, which may increase the rate of rape, expresses disrespect for women, and demonstrates a significant character defect.

Does your Real Doll Robot offer the option for non-consensual sex? What is your view on Robert Sparrow's argument? Is having sex with a robot nothing else than masturbation with a machine? Do your customers relate to an actual "somebody"?

We wouldn't purposely design a robot to engage in this type of scenario. The dolls, and the robot are best viewed as surrogate companions, or surrogate sex partners. Not rape training aides.

If your robot has the ability to adapt to the user then users could train the robot to show non-consensual sexual behavior, similar to how Microsoft's Tay chatbot was trained to tweet racist and xenophobic epithets. If the robot does not adapt then the user might become bored of its limited interaction patterns. How do you approach this dilemma?

The AI can easily have limits to that adaptation. I would rank it pretty high on our list to have such fail safes in the code. In fact, we would rather sacrifice some of the AI's ability to adapt than allow for such usage.

\footnotetext{
${ }^{1}$ https://campaignagainstsexrobots.org
}

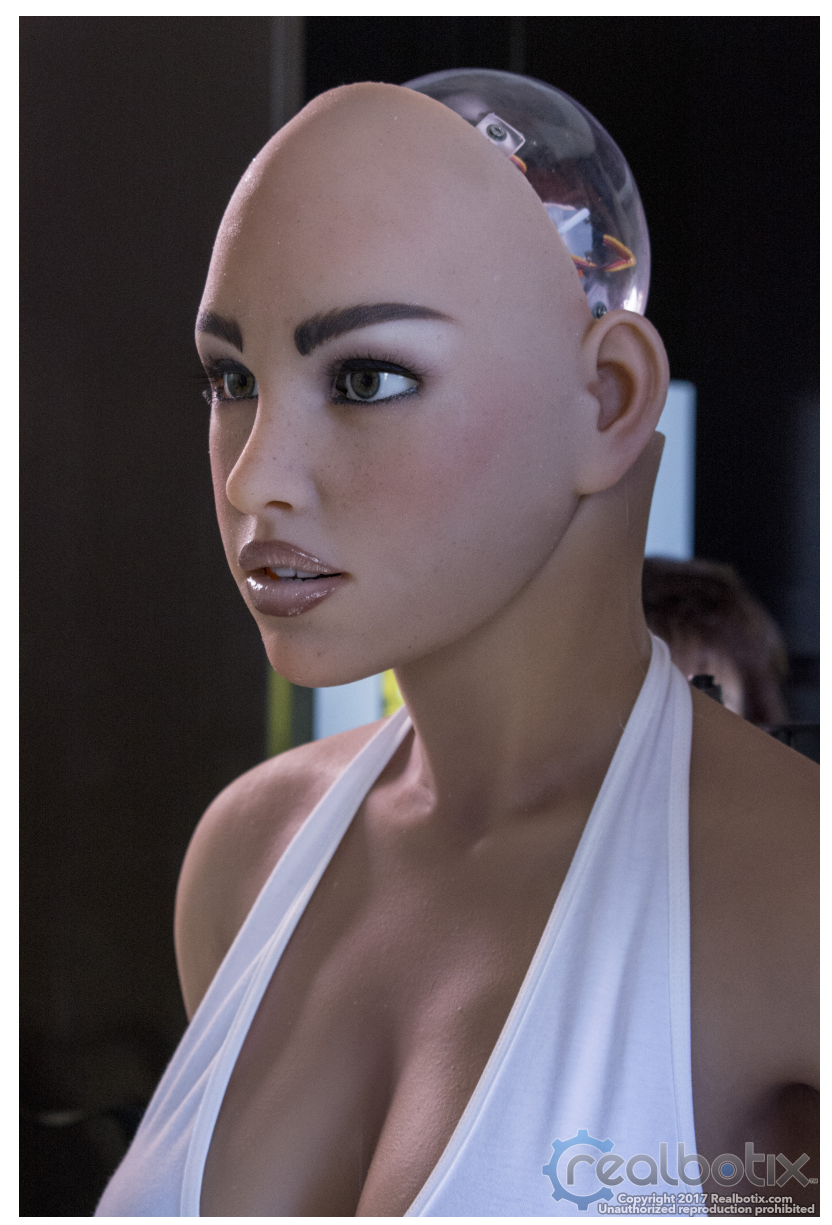

Figure 3: Harmony

I'd also like to add on this topic that I personally believe that behaviours such as rape and arousal from non consensual acts are likely genetic or at least heavily influenced by DNA, similar to those who are more likely to have addiction problems, or the presence of aggression or mental illness; That is to say, people who are predisposed to these behaviours might be born that way, and if they aren't predisposed to these behaviours I find it highly unlikely that a robot programmed to not consent to a sex act would create, or encourage such behaviour in an individual. The psychological factors that occur with these behaviours are likely dependant on the presence of a non-consenting human being; An act of domination over another person. I donâĂŹt logically see a robot filling that role effectively. Beyond that, for the sake of argument and discussion, wouldn't it be preferable that such an individual enact these behaviours with a doll or robot verses a human being? Couldn't a carefully programmed AI be used as a means of treating such behaviours in a therapeutic way? Or at the very least understanding them so that treatments can occur?

A similar ethical discussion is going on about violent computer games for already much longer. Does playing first person shooter games influence the real world behaviour of the players. There does seem to be a very vivid discussion, but empirical evidence does not 
seem to be conclusive yet. A very similar discussion about sex robots is almost unavoidable although empirical data will be harder to obtain since only few have had the opportunity to have actual sex with a robot yet. Give the experience with ethical discussion on violent computer games, how would you like to steer the discussion on sex robots into a productive direction?

Firstly, I think it is vitally important that we have an open mind when having any such discussion. Opinions based on fears and conjecture instead of scientific data should be viewed as just that, opinions. I do not feel that we are yet able to form reasonable assumptions on much of this because no one yet has a sex capable robot. I would gladly participate in any study that could give us insights into the repercussions or possible benefits of these new technologies. I think some of the radical fear based opinions might be swayed in a more reasonable direction if real data was available.

Human sexuality is a highly complex phenomena integrating physical stimulation, various hormones, and psychological states of mind. A wrong utterance can kill the mood in a second. Human robot sexual interaction can therefore be considered one of the most difficult tasks. On the other hand, rather cruel sex toys are currently being successfully used. This may lead to the expectation that human robot sexual interaction might be particularly easy. In your view, what are the best strategies for a robot to provide a satisfying sexual encounter?

This will be an ongoing process figuring this out. But I do believe that there will be a healthy amount of imagination involved for it to create a believable simulation. I also think that those who are able to differentiate that it is a robot rather than pretending it is a person, and accept that, or even enjoy that, will have much better experiences.
In your development, are you using a user-centered design process consisting of several cycles of development and testing? What methods and procedures have been helpful in the development of the robot?

We are still early in the real-world-testing phase. In truth, the early adopters will likely provide us with an enormous wealth of information and suggestions for improvements. To this point, we have Harmony interact with many people, both possible clients and friends, staff etc., and gauge their reactions.

The company behind Real Doll is called Abyss Creations LLC. This does some like a Cassandrian name. Are you going to lead humanity into the Abyss? If sex with a robot will become much more enjoyable than sex with a human partner, will humanity stop reproducing?

I can see where you might have wondered about the company name; However, the name was chosen based on where it would appear alphabetically in exhibitor listings. Sorry there isn't a deeper meaning.

\section{REFERENCES}

[1] Robert Sparrow. 2017. Robots, Rape, and Representation. International fournal of Social Robotics 9, 4 (01 Sep 2017), 465-477. https://doi.org/10.1007/ s12369-017-0413-z

[2] Kazuyoshi Wada and Takanori Shibata. 2007. Living with seal robotsâĂ ̌its sociopsychological and physiological influences on the elderly at a care house. IEEE Transactions on Robotics 23, 5 (2007), 972-980. https://doi.org/10.1109/TRO. 2007.906261 